\title{
New input circuits used for parameters determination of circuit breaker voltage and current paths
}

\author{
A. Lisowiec ${ }^{1}$, A. Nowakowski ${ }^{1}$ \\ ${ }^{1}$ The Research Centre for IT Systems and Hardware Applications \\ Tele and Radio Research Institute \\ Ratuszowa 11, 03-450 Warsaw (Poland) \\ Phone: +48 226192241 ext. 286, Fax number:+48 2261929 47, e-mail: aleksander.lisowiec@itr.org.pl, \\ andrzej.nowakowski@itr.org.pl
}

\begin{abstract}
In the article new constructions of voltage and current transducers have been presented that are used for parameters determination of current and voltage signals in the line protected by circuit breaker. These transducers are used with protection relay that is also a subsystem of source data acquisition for multi-criteria method of circuit breaker diagnosis. Because of the multifunctionality of the device the transducers have to work with, they have to be very accurate, have wide bandwidth and be stable over varying environment conditions like temperature and humidity. As current transducers, Rogowski coils have been described. They have been made with the use of multilayer PCB technology to achieve very high repeatability of coils parameters, low labor costs as well as high bandwidth and excellent linearity. The designs of Rogowski coils have been presented together with measurement results. As voltage transducers, new constructions of reactance transducers, resistive transducers and air core transformers have been presented. The main design effort has been put into achieving wide bandwidth and good linearity. In the paper input circuits adapted to new types of voltage transducers have been described as well as measurement results.
\end{abstract}

\section{Key words}

voltage transducer, current transducer, circuit breaker

\section{Introduction}

Circuit breaker diagnosis necessitates the determination with great accuracy and resolution $(100 \mu \mathrm{s})$ the timing parameters of circuit breaker like opening time and simultaneity between phases. Timing parameters are determined from phase current and voltage measurements. The frequency bandwidth of current and voltage paths should equal at least $8 \mathrm{kHz}$. While achieving such bandwidth in electronic amplifiers and analogue to digital converters is easy, traditional voltage and current core transformers used in power industry have very limited bandwidth, often below $1 \mathrm{kHz}$. Therefore for circuit breaker diagnosis it was necessary to develop new types of voltage and current transducers characterized by wide frequency bandwidth as well as good accuracy.

\section{Current transducers}

For current measurements Rogowski coils may be used. They have been used for many years in applications requiring measurements of large current in wide frequency bandwidth. The technologies used for making such coils were characterized by large man labour. Research work has been carried out at ITR to develop innovative technologies enabling to produce the coils with high parameters repeatability. These technologies are based on multilayer PCB. The coil is constructed from multilayer boards attached to a base board which provides mechanical support and connects all boards together electrically. On each layer of the multilayer board there is a basic coil in the form of a spiral. The coils on neighbouring boards are connected by buried and through vias. The design of two such coils is presented in fig. 1.

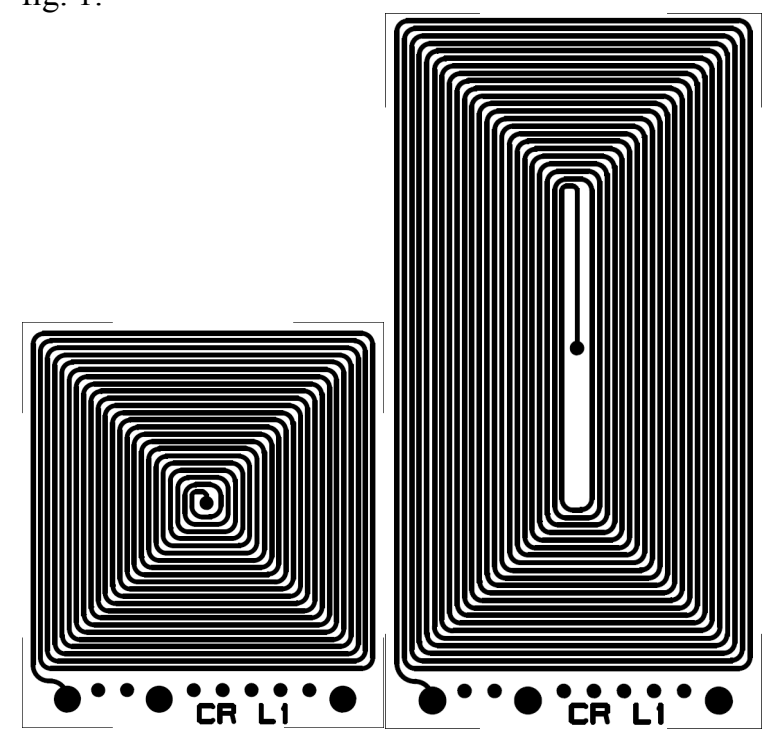

Fig. 1 Printed circuit coil designs

The main cost of the coil is the manufacturing cost of multilayer boards with printed circuit basic coils. 
Several of the designs of the Rogowski coils with 16layer base boards are presented in fig. 2 .

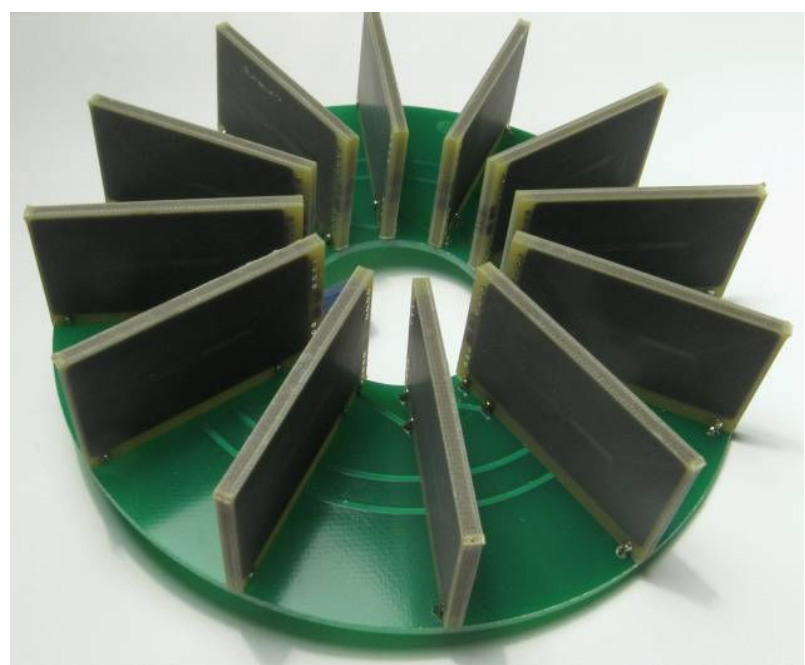

a) $\mathrm{CRH}$

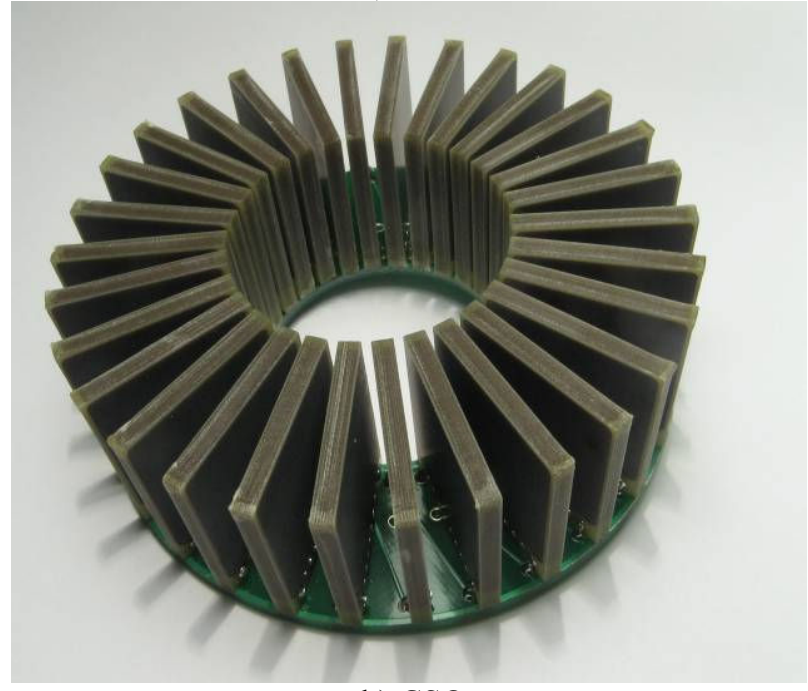

b) CSQ

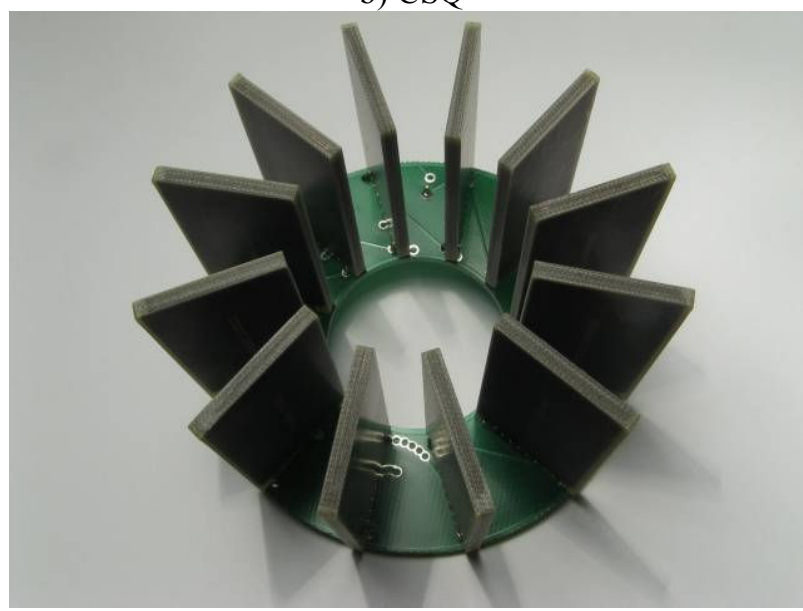

c) CRV

Fig. 2 Various design of Rogowski coils with 16-layer base boards

Fig. 3 shows a Rogowski coil design with 8layer base board and the same coil immersed in an epoxy resign.

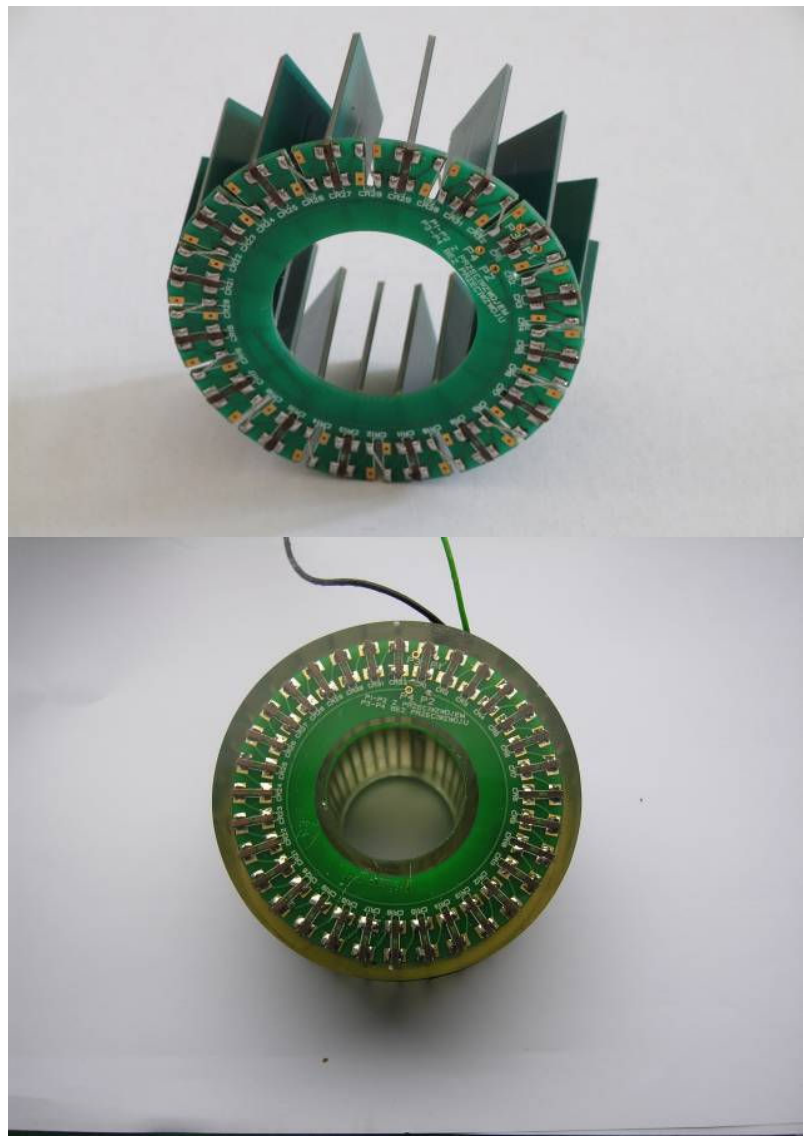

Fig. 3 A design of Rogowski coil with 8-layer base board

In applications requiring high measurement bandwidth it is necessary to determine the values of the elements of the equivalent electrical circuit of Rogowski coil, presented in fig. 4 . The value of $\mathrm{R}$ can be measured directly. The values of other element can be determined from the response of the coil to the current step which has the form shown by formula (1), where U1 is proportional to the derivative of the current.

$\mathrm{L} \quad \mathrm{R}$

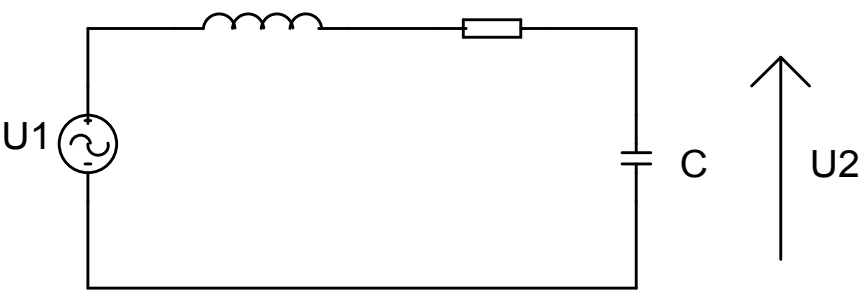

Fig. 4 An electrical equivalent circuit of Rogowski coil

$$
\mathrm{U} 2=\mathrm{U} 1 \cdot A \cdot e^{-\frac{t}{\tau}} \cdot \sin \left(\omega_{0} \cdot t\right)
$$

The parameters of the electrical circuit of the coils shown in fig. 2 are shown in table 1 , where $Q$ is the quality factor, $\xi$ is the damping ratio and $R_{k r}$ is the so called critical resistance. It is the load resistance with which the response of the coil to the current step does not show oscillatory character. The frequency characteristic of one of the Rogowski coils is shown in fig. 5 with load resistance (denoted by Robc in fig. 5) equal aproximately to the $R_{k r}$ and with load resistance much higher than $R_{k r}$. 
Table 1 Equivalent circuit parameters of selected Rogowski coils

\begin{tabular}{|c|c|c|c|c|c|c|c|}
\hline & $\begin{array}{c}R \\
{[\Omega]}\end{array}$ & $\begin{array}{c}L \\
{[\mathrm{mH}]}\end{array}$ & $\begin{array}{c}C \\
{[\mathrm{pF}]}\end{array}$ & $f_{0}[\mathrm{kHz}]$ & $Q$ & $\xi$ & $\begin{array}{c}R_{k r} \\
{[\mathrm{k} \Omega]}\end{array}$ \\
\hline $\mathrm{CRH}$ & 802 & 11.18 & 43.63 & 227.863 & 20 & 0.025 & 7.8 \\
\hline CRV & 762 & 7.9 & 73.47 & 208.884 & 13.6 & 0.037 & 5 \\
\hline CSQ & 1000 & 19.77 & 64.32 & 141.138 & 17.5 & 0.029 & 8.5 \\
\hline
\end{tabular}

The sensitivities of the coils are shown in table 2.

Table 2 Sensitivities of selected Rogowski coils

\begin{tabular}{|l|c|c|c|}
\hline Coil symbol & CRH & CRV & CSQ \\
\hline Sensitivity & $1.4 \mathrm{mV} / \mathrm{A}$ & $3 \mathrm{mV} / \mathrm{A}$ & $3.5 \mathrm{mV} / \mathrm{A}$ \\
\hline
\end{tabular}

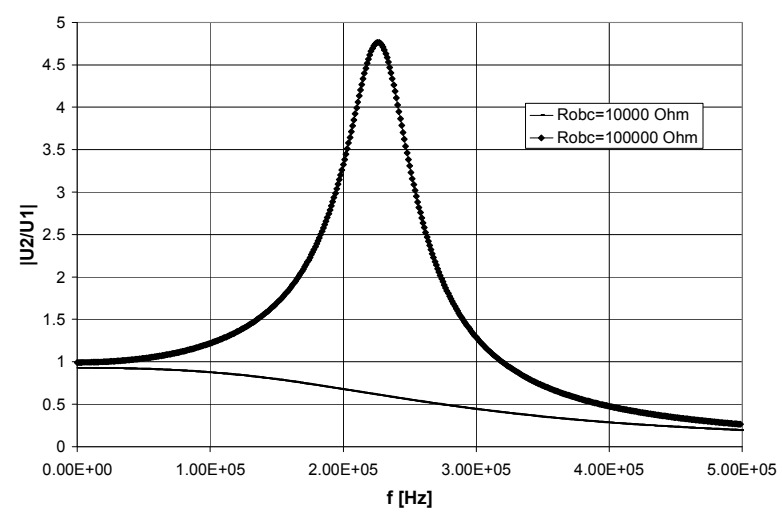

Fig. 5 Frequency characteristic of a Rogowski coil with two values of load resistance.

\section{Voltage transducers}

For voltage measurement in wide frequency bandwidth, reactance dividers, resistive dividers and air core transformers can be used. The research work has been carried out to develop and compare the parameters of transducers of the above mentioned types.

\section{A. Air core transformers}

The voltage transducer made as air core transformer must be characterized by low main inductance which makes it impossible to connect it directly to MV line. In order to limit the current flowing through primary winding it is necessary to use additional elements connected in series with primary winding. These can be resistors or capacitors. The design goal was to develop an air core transformer with output voltage equal to $200 \mathrm{mV}$ at input current not larger than $1 \mathrm{~mA}$. These conditions result from the necessity to achieve the necessary accuracy (min. 1\%) and permissible power dissipation within the transducer.

First designs used Rogowski coils connected as shown in fig. 6. One of the coil forms a primary winding and the other forms secondary winding. This construction exibits however very low mutual inductance which results in low output voltage.

One of the design objectives that were not easy to achieve wee sufficient isolation between layers and precise and durable boards connection.

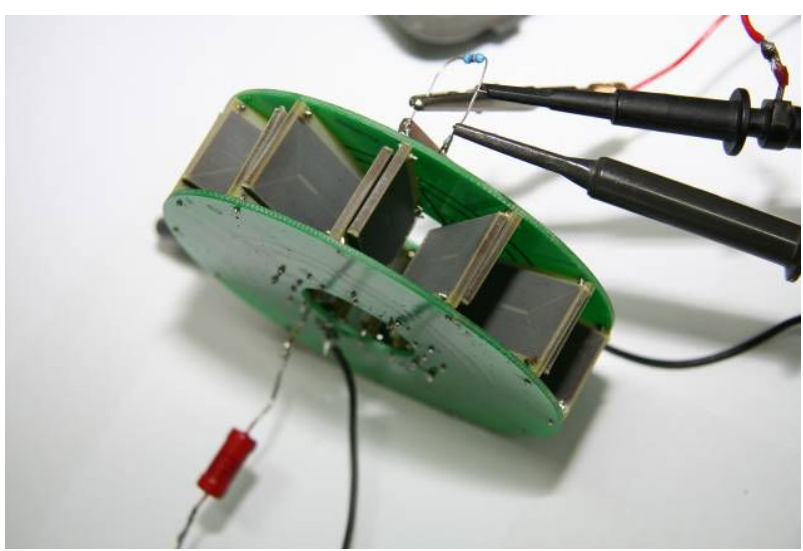

Fig. 6 An air core transformer made from two Rogowski coils

The finaly developed construction consists of alternately placed printed circuit boards forming primary and secondary transformer windings respectively. Each boared is made up of several layers containing printed coils in the form of spirals. The coils on consecutive layers are conneted in a way that preserves the turns directions. The boards are placed with centers aligned (fig. 7) and as close to each other as possible forming a sandwich with two secondary winding boards between every primary winding board.

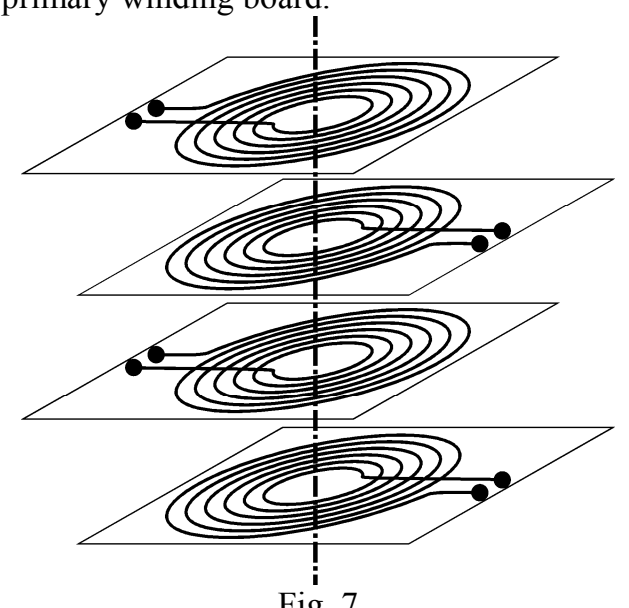

Fig. 7

The laboratory model of one of the final designs has been shown in fig. 8 .

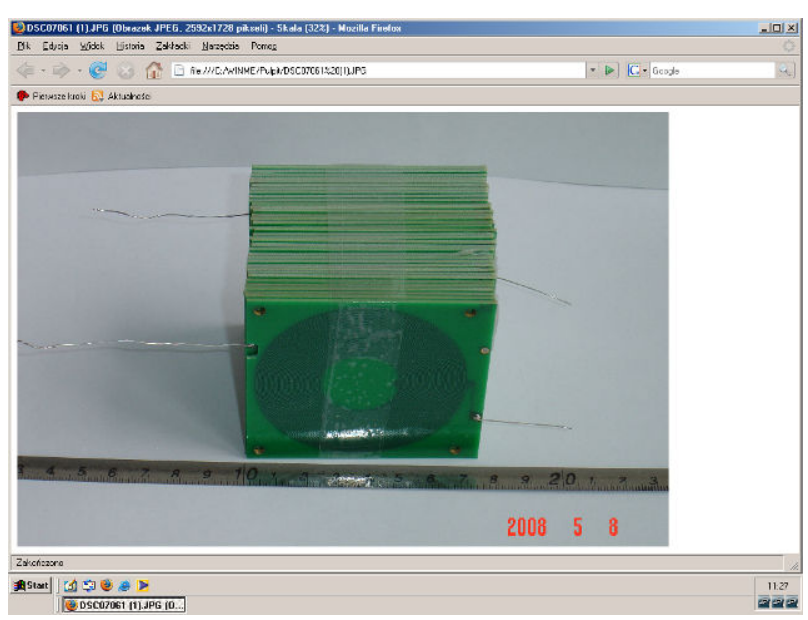

Fig. 8 Laboratory model of the final design of an air core transformer 
The simplified diagram of air core transformer connection in a measurment circuit is shown in fig. 9 . The developed air core transformers have been tested with resistive and capacitive divider.

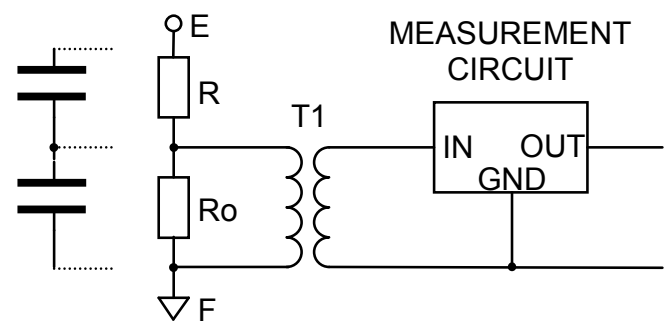

Fig. 9 An air core transformer connection in a measurement circuit

With final design the current of $1 \mathrm{~mA}$ flowing through primary circuit results in $141 \mathrm{mV}$ output signal which almost meets the design goal.

\section{B. Reactance transducers}

The main technical problems that have to be overcome while designing reactance transducers are phase shift between output signal and high tension input voltage, ferroresonance phenomenon and the necessity to employ voltage protection.

All reactance transducers require ferroresonance damping because the capacitance of the divider in series with the transformer inductance forms a resonance circuit. Due to various line interferences this circuit can resonate with higher line harmonics and cause core saturation. The magnetizing current can grow to such an extent that the electromagnetic part of the transformer may overheat. The voltage amplitude may become high enough to disrupt the isolation. In order to quickly damp the oscillations it is necessary to "get rid" of the energy stored in the transformer. It is achieved by connecting in series with the transformer winding a damping resistor through a resonance circuit tuned to line frequency.

The reactance transducers with transformer load suffer from nonlinearity of the transformer magnetizing curve.

The circuit for connecting the reactance transducer has been shown in fig. 10.

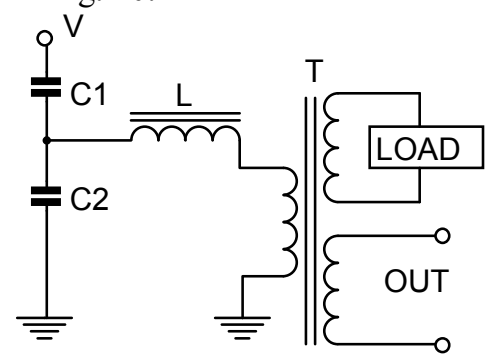

Fig. 10 Capacitive transducer connection

The large value of $\mathrm{C} 1$ - several tens of $\mathrm{nF}$, enables the voltage transformer $\mathrm{T}$ to draw the current of several tens of $\mathrm{mA}$ which makes it possible for it to work in linear region of its magnetization characteristic. To compensate for the phase shift a choke L is used. The choke, together with $\mathrm{C} 1 / \mathrm{C} 2$, forms resonance circuit, which represents pure resistance when tuned to the line frequency. In this case the phase shift is compensated. The value of $\mathrm{L}$ is selected individually for each transducer. For $1 \mathrm{nF} / 100 \mathrm{nF}$ capacitive divider a $100 \mathrm{H}$ choke is needed to form $50 \mathrm{~Hz}$ resonance circuit. The compensation scheme is effective only over very narrow frequency range so the reactance transducer is not suitable in situations where signals with high harmonic content are to be measured accurately.

Fig. 11 shows a laboratory model of capacitive $10 \mathrm{nF} / 1 \mu \mathrm{F}$ voltage divider.

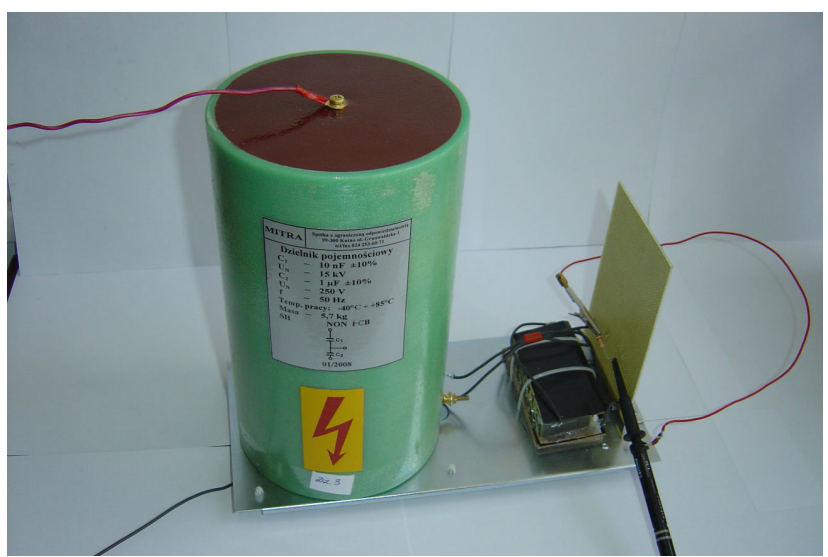

Fig. 11 A laboratory model of $10 \mathrm{nF} / 1 \mu \mathrm{F}$ voltage divider

\section{Resistive transducers}

Resistive transducers have much higher accuracy and temperature stability than capacitive dividers and they do not introduce the phase shift. The problems with their application are power dissipation and the absence of galvanic isolation. Modern measurement circuits require very low input currents, so by employing very high value resistors it is possible to eliminate the problem of power dissipation in the divider. The galvanic isolation problem is much harder to solve. The most common solution is to use separation transformer. The main disadvantage of such a solution is the introduction of phase shifts and nonlinearity because of core saturation. The combination of resistive divider with an air core transformer is not feasible because small efficiency of the air core transformer requires the use of relatively large primary current. A solution that sidesteps the galvanic isolation problem is to connect the resistive dividers in such a way that a virtual zero point is created and part of the measurement circuit can be connected to this virtual zero.

The resistors in the laboratory model of high voltage divider are made from nickel-chromium paste on ceramic substrate. Such solution quaranties constant resistance value over large power and temperature range as well as stability in time. Both resistors are manufactured on the same ceramic plate, tirmmed with laser and immersed in epoxy resin.

The divider parameters that have been achieved are presented below:

working voltage $-75 \mathrm{kV}(90 \mathrm{kV} / 15 \mathrm{~min} ., 110 \mathrm{kV}$ peak $)$, divider power $\quad-\quad 30 \mathrm{~W}$, resistance $\quad-\quad 300 \mathrm{M} \Omega$, dividing ratio division accuracy division stability working temperature range $1: 16000$ $0.1 \%$, $10 \mathrm{ppm} /{ }^{\circ} \mathrm{C}$, 10 to $70{ }^{\circ} \mathrm{C}$ 


\section{Higher harmonics transfer characteristic}

This test has been carried out with low value input voltage measurment setup. The measurement voltage was fed to the input of the divider under test through $100 \mathrm{k} \Omega$ resistor.

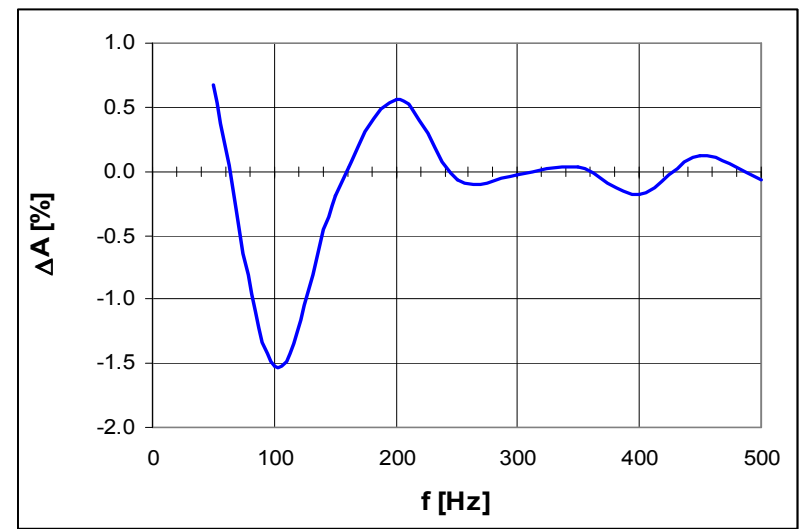

Fig. 12 Relative error of the transfer function versus frequency.

\section{Measurement circuit}

A modern system for line voltage and current signals measurement is shown in fig. 13. Current signals are measured by Rogowski coils and voltage signals by resistive dividers. The output signals of these transducers are fed directly to the blocks labelled SIGNAL CONDITIONING that filter the input signals to prevent aliasing and amplify (or damp) them to the level appropriate for multichannel analog to digital converter MDAC. The integration of Rogowski coils output signals is being carried out digitally by signal processor.

The elimination of additional current and voltage transducers at the input of the measurement device enables the application of special diagnostic procedures that test the complete current and voltage signals measurement paths.

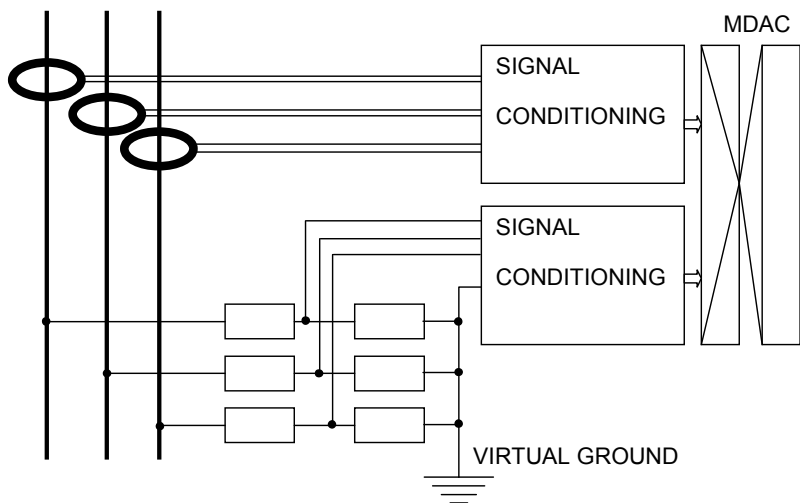

Fig. 13 A modern line voltage and current signals measurement system with Rogowski coils and resistive transducer

\section{Conclusion}

Unconventional voltage and current transducers give the possibility of designing modern, high precision measurement circuits for MV equipment. Air core transducers have been used for some time but designs used so far had some flaws. The transducer, developed at ITR whose design makes use of PCB technology has high precision, repeatability and sensitivity. The main disadvantage of an air core transformer is the frequency characteristic which is not flat over frequency range up to $2 \mathrm{kHz}$. Further research work in this direction is being carried out.

The most promising design for voltage measurement is a resistive divider which is naturaly wide band, has high stability and keeps its parameters over wide temperature range. Disadvantages that have limited so far the application of resistive transducers, namely high dissipated power and lack of galvanic isolation have been overcome by the introduction of virtual ground point.

\section{Acknowledgement}

The research presented in the paper is a part of a development project cofounded by the European Union within European Union Structural Funds, POIG (Operational Programme Innovative Economy), project number WND-POIG.01.03.01$14-141 / 08$

\section{References}

[1] A. Nowakowski, J. Borczyński, "Medium Voltage Measurement Circuit With Air Core Transformer", Elektronika, (12/2008), pp. 44-48 (in Polish).

[2] A. Nowakowski, G. Kowalski, A. Kalinowski, "Research On Reactance Transformers For Use In Power Measurement In Medium Voltage Network", Elektronika, (12/2008), pp. 39-43 (in Polish). 